\title{
Cryptococcal Meningitis in an Immunocompetent: A Rare Case Report
}

\author{
Kumar A* \\ Department of Microbiology, The Mission Hospital, India
}

*Corresponding author: Avinash Kumar, Consultant, Department of Microbiology, The Mission Hospital, Bidhanagar, Durgapur-713212, West Bengal, India, Tel: 8802469796; Email: lakchya@gmail.com

\section{Case Report}

Volume 4 Issue 2

Received Date: July 14, 2020

Published Date: August 10, 2020

DOI: $10.23880 /$ jidtm- 16000141

\section{Abstract}

Cryptococcal meningitis used to be a disease of immunocompromised patient but, in today's scenario, it is also infecting immunocompetent individuals. It usually affects immunocompromised patients. Here we present a case report of cryptococcal meningitis in an immunocompetent individual of 54 years.

Keywords: Crypyptococcal meningitis; Immunocompromised; Immunocompetent; Cryptococcus neoformans; Indian ink

Abbreviations: CM: Cryptococcal Meningitis; ELISA: Enzyme-Linked Immunosorbent Assay; HIV: Human Immunodeficiency Virus; AIDS: Acquired immunodeficiency syndrome; CSF: Cerebrospinal Fluid.

\section{Introduction}

Cryptococcus neoformans (C.neoformans), an encapsulated yeast, is the etiology of Cryptococcal meningitis (CM). It is commonly the first opportunistic infection to be diagnosed, in patients with HIV/AIDS. The mode of transmission of CM is usually inhalation of spores of fungus, which spread systemically via the lungs. Meningitis is most common presenting infection than pneumonia, skin infections, and osteomyelitis [1]. C. neoformans is a ubiquitous environmental fungus and a fatal opportunistic infection. CM draws more and more clinician's attention due to enhancement of the relevance ratio and recognition of its life-threatening effect. There are confirmed official statistics that approximately one million new cases of $\mathrm{CM}$ occur each year worldwide with more than 50 percent fatality [1].

The economic and medical burden in sub-Saharan Africa and Southeast Asia is due to towering morbidity and a requirement of long-term management [2]. C.neoformans is frequently found in pigeon feces, soil, milk and human being's oral cavity [3]. The C.neoformans usually invades through respiratory tract of the immunocompromised hosts and damaged skins by the exposure of pigeon feces, leading to the varied infection: pulmonary cryptococcosis, skin nodules, cryptococcal meningitis, Cryptococcus bacteremia, etc. The method by which particularly cryptococcal infection occurs in immunocompetent hosts is not fully understood, but disease in these individuals may result due to high level of organism exposure, exposure to a pathogenic, cryptococcal strain or subtle or undetectable immune deficit in the host $[4]$.

\section{Case History}

A 54 year old hypertensive, non-diabetic male, farmer by occupation, admitted with complains of headache, vomiting and weakness for 1 month. On physical examination, body temperature was 39.3 degree centigrade, blood pressure $128 / 82 \mathrm{mmHg}$ and a regular pulse of 82 beats/min. The color of his mucous membrane and skin were normal without rashes or herpes. Systemic examination of heart, lungs and pupillary light reflex were normal. Babinski's sign and Kerning's sign were positive with stiff-neck. The patient was admitted in ICU for management. CSF examination (Table 1) was done after lumbar puncture. The patient was found to be serologically non-reactive to HIV, HCV and HBV 
(Table 1). All investigations were within normal limit except CSF examination which showed encapsulated budding yeast cells with daughter cell suggestive of Cryptococcus spp. by Indian ink microscopy (Figure 1). On gram stain microscopy of CSF, gram positive budding yeast cells with capsule seen suggestive of cryptococcus spp. Cryptococcus neoformans was isolated after 72 hrs of aerobic incubation at 37 degree centigrade from Sabourauds Dextrose agar media (Figures 2 $\& 3)$. The Cryptococcus neoformans was found to be urease positive also (Figure 4).

\begin{tabular}{|c|c|c|}
\hline Microbiology & Biochemistry & Cytology \\
\hline $\begin{array}{c}\text { Gram stain-Few pus cells seen, Moderate Gram positive oval to spherical } \\
\text { single and budding yeast cells with daughter cells seen suggestive of } \\
\text { Cryptococcus spp. }\end{array}$ & Sugar-20mg/dl & Total count-3/cmm \\
\hline Ziehl Neelson stain-No AFB seen & Protein-59.7mg/dl & RBC-Nil \\
\hline $\begin{array}{c}\text { Indian Ink stain-Encapsulated single and budding yeast cells with daughter } \\
\text { cells seen }\end{array}$ & ADA-20.19 & Neutrophil-00 \\
\hline $\begin{array}{c}\text { Culture-Cryptococcus neoformans isolated after 72 hrs of aerobic } \\
\text { incubation at 37 degree centigrade }\end{array}$ & & Lymphocytes-100\% \\
\hline
\end{tabular}

Table 1: CSF Examination.
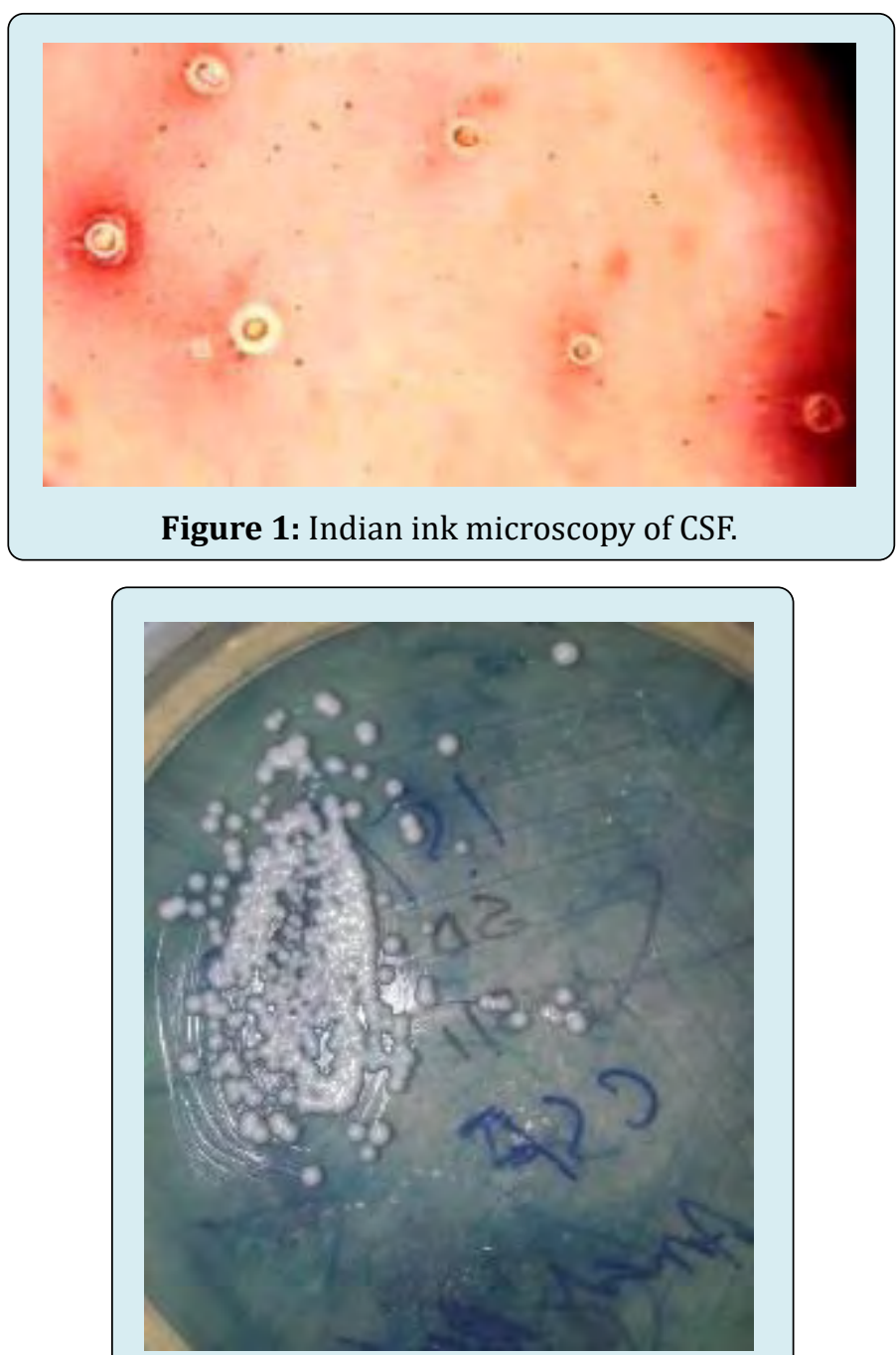

Figure2: Cryptococcus isolated in SDA.

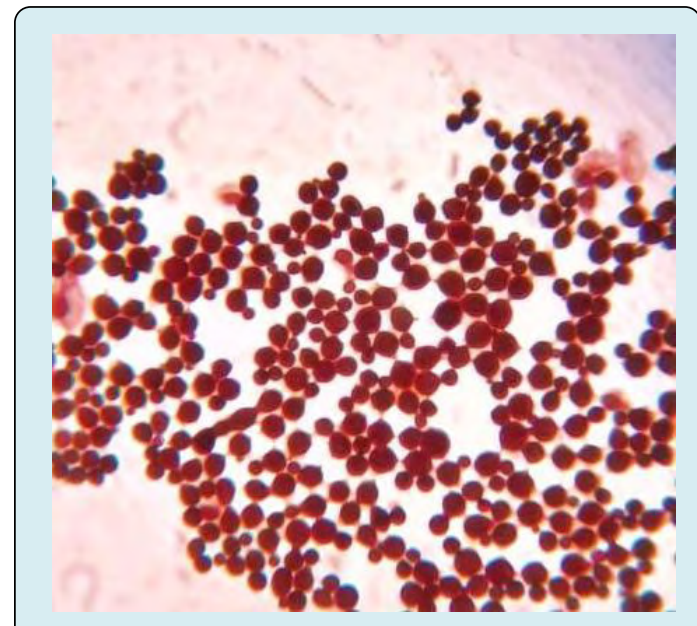

Figure 3: Gram stain of fungal culture.

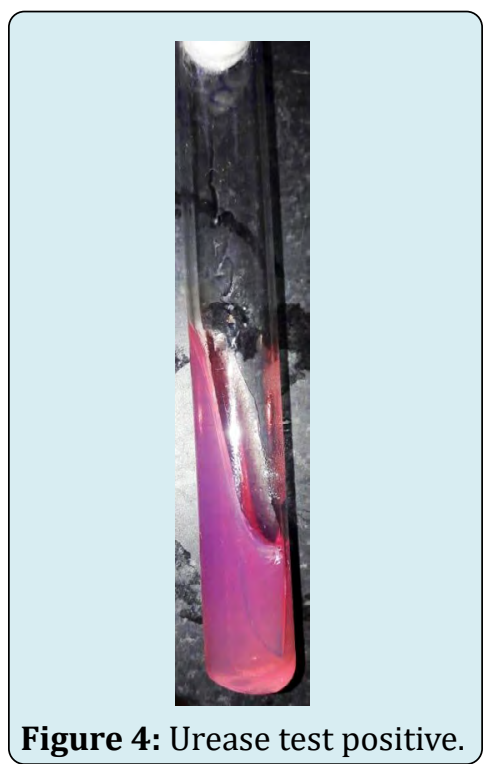

Kumar A. Cryptococcal Meningitis in an Immunocompetent: A Rare Case Report. J Inf Dis Trav Med 2020, 4(2): 000141. 


\section{Discussion}

The patient in our case is a farmer by occupation, so respiratory mode may be the mode of infection. Although $\mathrm{CM}$ is rare among immunocompetent patients, the delayed diagnosis and suboptimal treatment surely may result in poor prognosis and high mortality. So, the importance of clinical suspicion for cryptococcal infection in immunocompetent patients with subtle clinical presentation is very important. Laboratory services play an important part in addition to clinical suspicion in this type of meningitis. The CSF examination should be done with latex agglutination test, along with gram stain and Indian ink microscopy. For rapid diagnosis, antigen testing is found to be more sensitive than culture, and both latex agglutination assay and ELISA (enzyme-linked immunosorbent assay) testing have sensitivity and specificity $>90 \%$, though sensitivity is felt to be lower for non-HIV-infected individuals and should not be used to rule out disease [5]. Current published literatures recommend new diagnostic method-lateral flow immunochromatographic assay (LFA), which is more costeffective, requiring a drop of blood or CSF, only taking ten minutes to report and can be shipped at room temperature [6].

Immunocompromised patients with underlying diseases, such as HIV, solid organ transplantation, liver cirrhosis, tuberculosis, SLE and other diseases which need the long-term therapy of immunosuppressive agent are frequently caused by C.neoformans [1,3]. Although genetic deficits, medications, malignancy and AIDS usually causes immunosuppression, but important to consider that several states notably diabetes mellitus, alcoholism, cirrhosis, and autoimmune condition may precipitate immunosuppression, potentially predisposing hosts to opportunistic infection. When immunocompetent patient manifests with headache, fever and other altered mental status than viral or bacterial meningitis can be misdiagnosed very easily [7].

However, CM in an immunocompetent host carries mortality of $12 \%$ and is often delayed in presentation due to the frequently subacute nature of symptom development [8]. Therefore, the importance of clinical history and quality laboratory services make a key role in diagnosing cryptococcal meningitis at the earliest to reduce the mortality. Antifungal agents and control of intracranial pressure should be started earliest to avoid morbidity and mortality, as well as to control underlying causes of potential immunosuppression [9].

\section{References}

1. Lui G, Lee N, Ip M, Choi KW, Tso YK, et al. (2006) Cryptococcosis in apparently immunocompetent patients. QJM 99(3): 143-151.

2. Dixit A, Carroll SF, Qureshi ST (2009) Cryptococcus gattii: An emerging cause of fungal disease in North America. Interdiscip Perspect Infect Dis 2009: 840452.

3. Aberg JA, Mundy LM, Powderly WG (1999) Pulmonary cryptococcosis in patients without HIV infection. Chest 115(3): 734-740.

4. Brouwer AE, Siddiqui AA, Kester MI, Sigaloff KCE, Rajanuwong A, et al. (2007) Immune dysfunction in HIV-seronegative,Cryptococcus gattii meningitis. J Infect 54(3): 165-168.

5. Williamson PR, Jarvis JN, Panackal AA, Fisher MC, Molloy SF, et al. (2017) Cryptococcal meningitis: epidemiology, immunology, diagnosis and therapy. Nat Rev Neurol 13(1): 13-24.

6. Kabanda T, Siedner MJ, Klausner JD, Muzoora C, Boulware DR (2014) Point-of-care diagnosis and prognostication of cryptococcal meningitis with the cryptococcal antigen lateral flow assay on cerebrospinal fluid. Clin Infect Dis 58(1): 113-116.

7. Zhu LP, Shi YZ, Weng XH, Müller FMC (2002) Case Reports. Pulmonary cryptococcosis associated with cryptococcal meningitis in non-AIDS patients. Mycoses 45(3-4): 111-1117.

8. Pappas PG, Perfect JR, Cloud GA, Larsen RA, Pankey GA, et al. (2001) Cryptococcosis in human immunodeficiency virus-negative patients in the era of effective azole therapy. Clin Infect Dis 33(5): 690-699.

9. Day JN, Chau TTH, Wolbers M, Mai PP, Dung NT, et al. (2013) Combination antifungal therapy for cryptococcal meningitis. N Engl J Med 368(14): 1291-1302. 